\title{
Matching of Stereo Curves - A Closed Form Solution
}

\author{
Yaonan Zhang and Jan J. Gerbrands* \\ Information Theory Group \\ Department of Electrical Engineering, Delft University of Technology \\ P.O. Box 5031, 2600 GA Delft, The Netherlands
}

\begin{abstract}
A method is described in this article to solve the stereo matching problem of general closed planar curves under possible imperfect segmentation and occlusions, provided that camera parameters are known. The method decomposes the parameters related to an object plane, i.e. slant, tilt and scale factor, and uses a histogram technique to estimate these parameters. The parameter estimation is based on the disparity information of the stereo curves. Point correspondence plays an important role in the method. We solve this problem in a dynamic programming style. The final matching is assessed by applying a distance transformation. The method has been applied successfully to several practical examples.
\end{abstract}

\section{Introduction}

In this paper, we present a method to solve the stereo matching problem of general closed planar curves, provided that camera parameters are known. The stereo curves are assumed to be the output of some segmentation procedures and represent the boundaries of certain objects. Usually, segmentation procedures are not perfect and occlusions may occur. That means that some portions of the stereo curves may be detected incorrectly or may be missing altogether. The general solution of this problem is not available. There are quite a number of publications dealing with curve-based matching [1-5]. Relevant surveys can also be found in $[6,7]$. The existing curve matching methods have the following problems: 1) some methods can only deal with two dimensional rotation, translation, and scaling, but cannot deal with perspective transformation; 2) some methods are sensitive to noise and cannot tackle errors due to bad segmentation and occlusion; 3) some methods have strict constraints with respect to the shape of the curves concerned, etc. In this article, we develop a curve matching method which is capable of dealing with perspective transformation and of handling possible errors due to bad segmentation and occlusion without making assumptions on the shape of the curves. The assumptions we do make in this paper are: 1) the stereo curves are the perspective projections of 3-D planar curves in an object space; 2 ) the stereo curves are closed; 3 ) any curve concerned

\footnotetext{
* This research is partially supported by the NWO/SION project "Model Based Image Sequence Analysis"
} 
is a simple curve (if a curve representing a region boundary stems from the output of a region growing algorithm, this requirement is alway guaranteed; if the curve is produced by other kinds of segmentation techniques, some operations may be needed in order to meet this requirement). The method we propose in this paper decomposes parameters related to an object plane, i.e. slant, tilt and scale factor, and uses a histogram technique to estimate these parameters based on disparity information. The point correspondence problem is solved in a dynamic programming style. The final matching is assessed by applying a distance transformation.

\section{The Method}

The merit of the overall matching between the stereo curves can be judged by the difference between the two normalized curves. By normalization in the stereo case, we mean that one curve is transferred before matching. Suppose we transfer the left curve into the right image and denote the transferred $x^{\prime}$ coordinate as $x^{*}$ (note that there is no difference on $y$ coordinates on a correctly matched point pair ${ }^{2}$ ).

We use the following formula for the geometric transformation:

$$
\left\{\begin{array}{c}
x^{*}=x^{\prime}+S_{c}\left(f_{c}+\tan (\Omega) \cos (\Gamma) x^{\prime}+\tan (\Omega) \sin (\Gamma) y^{\prime}\right) \\
\text { if } \Omega<45^{o} \\
x^{*}=x^{\prime}+S_{c}^{\prime}\left(\tan \left(\Omega^{\prime}\right) f_{c}-\cos (\Gamma) x^{\prime}-\sin (\Gamma) y^{\prime}\right) \\
\text { if } \Omega^{\prime} \leq 45^{o} .
\end{array}\right.
$$

In this equation, $x^{\prime}, y^{\prime}$ are $x, y$ coordinates of points along the left curve, $f_{\mathrm{c}}$ is the principal distance of the camera, the slant $\Omega\left(\Omega^{\prime}=90^{\circ}-\Omega\right)$, tilt $\Gamma$, and scale factor $S_{c}$ determine the orientation and distance of object plane corresponding to the stereo curves. The normal vector of the object plane is determined by three points $P_{1}, P_{2}, P_{3}$ or two vectors $\mathbf{V}_{\mathbf{1}}$ and $\mathbf{V}_{\mathbf{2}}$ on the plane. Let $\mathbf{n}=\left\{n_{x}, n_{y}, n_{z}\right\}$ be the normal vector, then $\Omega$ and $\Gamma$ can be calculated by

$$
\begin{gathered}
\Omega=\arccos \left(\frac{\left|n_{z}\right|}{\sqrt{n_{x}^{2}+n_{y}^{2}+n_{z}^{2}}}\right) \\
\Gamma=\arccos \left(\frac{\left|n_{x}\right|}{\sqrt{n_{x}^{2}+n_{y}^{2}}}\right) .
\end{gathered}
$$

Where

$$
\begin{aligned}
n_{x}= & \left\{\left(y_{1}^{\prime} d_{3}-y_{3}^{\prime} d_{1}\right)\left(d_{2}-d_{3}\right) f_{c}-\right. \\
& \left.-\left(y_{2}^{\prime} d_{3}-y_{3}^{\prime} d_{2}\right)\left(d_{1}-d_{3}\right) f_{c}\right\} L / d_{1} d_{2} d_{3}^{2}
\end{aligned}
$$

${ }^{2}$ We assume in this article that the stereo curves are rectified. 


$$
\begin{aligned}
n_{y}= & \left\{\left(x_{2}^{\prime} d_{3}-x_{3}^{\prime} d_{2}\right)\left(d_{1}-d_{3}\right) f_{c}-\right. \\
& \left.-\left(x_{1}^{\prime} d_{3}-x_{3}^{\prime} d_{1}\right)\left(d_{2}-d_{3}\right) f_{c}\right\} L / d_{1} d_{2} d_{3}^{2} \\
n_{z}= & \left\{\left(x_{1}^{\prime} d_{3}-x_{3}^{\prime} d_{1}\right)\left(y_{2}^{\prime} d_{3}-y_{3}^{\prime} d_{2}\right)-\right. \\
& \left.-\left(x_{2}^{\prime} d_{3}-x_{3}^{\prime} d_{2}\right)\left(y_{1}^{\prime} d_{3}-y_{3}^{\prime} d_{1}\right)\right\} L / d_{1} d_{2} d_{3}^{2} .
\end{aligned}
$$

In the above equations, $L$ is the length of the baseline; $\left(x_{i}^{\prime}, y_{i}^{\prime}\right)$ and $\left(x_{i}^{\prime \prime}, y_{i}^{\prime \prime}\right)$ are the photo coordinates of corresponding left and right image points on the stereo curves, respectively; and $d_{i}$ is the disparity value for a pair of points over the stereo curves.

$S_{c}$ is solved in the near-horizontal case $\left(\Omega<45^{\circ}\right)$ by

$$
S_{c}=-\frac{d}{f_{c}+\tan (\Omega) \cos (\Gamma) x^{\prime}+\tan (\Omega) \sin (\Gamma) y^{\prime}}
$$

In the case of near-vertical $\left(\Omega \geq 45^{\circ}\right)$, the scale factor is defined as $S_{c}^{\prime}$, and is calculated by

$$
S_{c}^{\prime}=-\frac{d}{\tan \left(\Omega^{\prime}\right) f_{c}-\cos (\Gamma) x^{\prime}-\sin (\Gamma) y^{\prime}}
$$

\section{The Algorithm}

The first step in our algorithm is to establish the point correspondence. Based on the disparity information, we can estimate the parameters for curve normalization for each three points along the stereo curves. The most likely values for the parameters are calculated by a histogram technique. After curve normalization, the differences between the two curves are measured using a distance transformation. The details of the algorithm are described below.

\subsection{Point correspondences}

The aim of the point correspondence is to establish the corresponding relationship between the points on the stereo curves. The algorithm we used is developed in a dynamic-programming style. Each point on the left curve is tested against a number of candidate points on the right curve. The final matches are the unique matches which minimize the sum of the disparity values.

\subsection{Weighting the curve direction}

The accuracy of the disparity measurement along the curve is quite dependent on the direction of the curve at a specific position. Obviously, the disparities of nearhorizontal segments are less reliable than the ones on near-vertical segments. They should be treated differently in the parameter estimation procedure. 


\subsection{Histogram analysis}

Histogram analysis is a useful technique for image processing to measure the relative frequency of occurrence of signal values within a certain range. The technique is used here as a kind of voting mechanism to select the most populous value for the estimated parameter. In our algorithm, a hierarchical histogram is actually adopted, which means that for each parameter, several levels of histograms are used, with the number of bins at each level fixed. The calculated parameter values are first put into corresponding bins at level 1 . After all values have been computed, the peak position at that level is detected. At the next level, the same parameter values are used, but the bin width is reduced. After the computations at several levels have been finished, the peak position at the final level is used to calculate the final parameter value. The reason for using this multi-level histogram is to avoid possible multiple peaks on the histogram and to get a more accurate peak position through the refinement of the levels.

\subsection{Distance transformation}

The final step of the algorithm uses the distance transformation and distance measurement techniques to measure the similarity between two shapes. The distance transformation converts the pixels of a curve into a gray-level image where all pixels have a value corresponding to the minimum distance to the curve.

\subsection{Matching measure}

In order to get a proper match measure, the searching curve is transferred into a distance image, the left normalized curve is then superimposed on this image. Defining the distance value at point $j$ on the reference curve as $D_{j}$, the good match points are detected by thresholding the $D_{j}$, i.e.

if

$$
D_{j}<\epsilon_{d}
$$

then point $j$ is a good match point. Here $\epsilon_{d}$ is the distance threshold.

Let $N_{c}$ be the total number of points on the reference curve, $N_{c}^{\prime}$ be the total number of points having good matching decided by Eq. (6), the match measure between the stereo curves are expressed by

$$
m_{s}=\frac{N_{c}^{\prime}}{N_{c}}
$$

Another way of defining matching measure is to consider the average value of $D_{j}$. But in case there are some errors caused by bad matches, the average distance value becomes unreliable. The definition by Eq. (7) reflects what fraction of the reference curve has been matched reliably. 


\section{Experiment}

We first show the results carried out on the stereo curves shown in Fig. 1 (the curves are highlighted in the figure). These stereo curves result from regionbased segmentation. Given these two curves (the left curve has 149 pixels and the right one has 199), our task is to match them based on the method described in the previous sections. The result for point correspondences shows that the big errors occur along near-horizontal segments. Consequently, the weight function is calculated based on the curve direction. To estimate the parameters slant and tilt, a three-level hierarchical histogram analysis was used. At each level, 8 bins are used, the population of a certain value is put into the corresponding bin. A peak is detected and is refined to the next level where the width of bin is reduced by half. The angle ranges of slant and tilt are both $90^{\circ}$ (estimated $\Gamma$ value from the histogram is in the first quadrant, and the real quadrant $\Gamma$ belongs to is decided by the signs of $n_{x}, n_{y}$, the finest bin on the last level of the histogram has width of $90 / 32=2.81250^{\circ}$. The width of a bin should not be too small otherwise there will be too many local peaks on the histograms. The final position of a peak can be improved by averaging the data positions within the bin of the detected peak. When calculating slant and tilt, a four-point interval is used to sample points on the disparity curve. In order to evaluate the match, the left curve is transferred into the right image by formula (1).

Another example is shown in Fig. 2. In this figure, there are some differences between the stereo curves produced by bad segmentation. From our many experiments, it follows that our method produces acceptable results.

\section{Conclusion}

In this paper, we described a closed form solution to the general stereo curve matching problem. Experiments have shown that the method is quite promising and is robust against imperfect segmentation and occlusion. The extension of this work is to consider other kinds of curves, for example, the curves on polynomial surfaces.

\section{References}

1. B. Bhanu and O. Faugeras, "Shape matching of two-dimensional objects," IEEE Transactions on Pattern Analysis and Machine Intelligence, vol. 6, pp. 137-156, 1984.

2. E. Salari and S. Balaji, "Recognition of partially occluded objects using B-spline representation," Pattern Recognition, vol. 24, pp. 653-660, 1991.

3. D. Sherman and S. Peleg, "Stereo by incremental matching of contours," IEEE Transactions on Pattern Analysis and Machine Intelligence, vol. 12, pp. 1102-1106, Nov. 1990.

4. A. Bruckstein, R. Holt, A. Netraval, and T. Richardson, "Invariant signatures for planar shape recognition under partial occlusion," CVGIP: Image Understanding, vol. 58, pp. 49-65, July 1993. 
5. Z. Pizlo, "Recognition of planar shapes from perspective images using contour-based invariants," CVGIP: Image Understanding, vol. 56, pp. 330-350, 1992.

6. B. Kamgar-Parsi, A. Margalit, and A. Rosenfeld, "Matching general polygonal arcs," CVGIP: Image Understanding, vol. 53, pp. 227-234, 1991.

7. E. Arkin, L. Chew, D. Huttenlocher, K. Kedem, and J. Mitchell, "A efficiently computable metric for comparing polygonal shapes," IEEE Transactions on Pattern Analysis and Machine Intelligence, vol. 13, pp. 209-216, 1991.

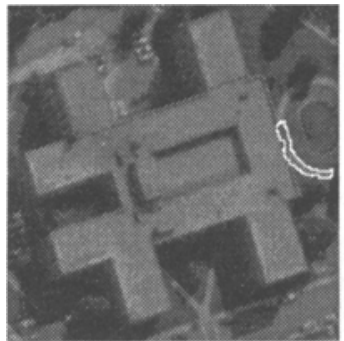

(a)

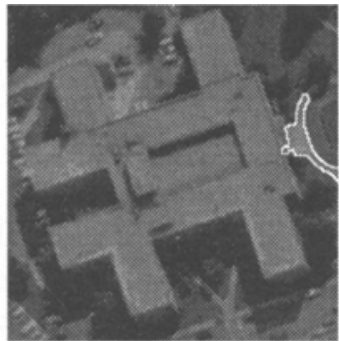

(b)

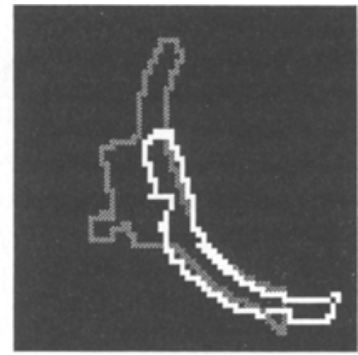

(c)

Fig.1. In this example, the left curve (a) has 149 points, and the right curve (b) has 199 points; (c) shows the normalized stereo curve. The final match measure is 0.46 .

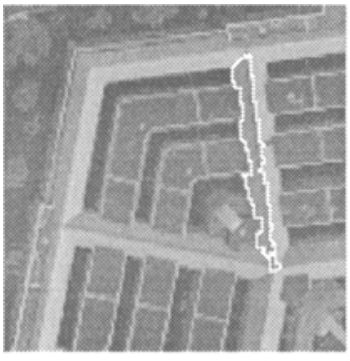

(a)

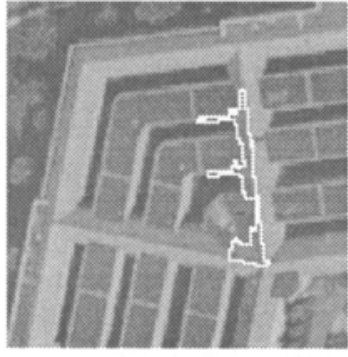

(b)

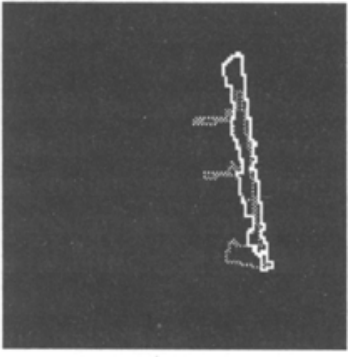

(c)

Fig.2. In this example, the left curve (a) has 223 points, and the right curve (b) has 259 points; (c) shows the normalized stereo curves. The final match measure is 0.42 . 